\title{
Design and Fabrication of Narrow Band Infrared Detector
}

\author{
Dr. Khalid Khalil Mohammad Omar Badir Mohammad \\ University of Mosul \\ College of Engineering \\ Electrical Department
}

\begin{abstract}
Most of infrared detectors used in various electrical equipments have wide spectral response and usually needs front filter to prevent visible light from passing to the detector. This research is intended to study and fabricate an indium doped silicon (n) (In-Si) structure to achieve a photo detector working as narrowband infrared detector. The SCAPS program was used as a simulation tool in order to achieve the optimum structure design. Then the (In-Si) infrared detector was fabricated using vacuum evaporation deposition technique. The fabricated photodiode shows a narrowband spectral response at $940 \mathrm{~nm}$. The results of the simulated and practical samples are comparable which gives a simple and cheap method for obtaining narrowband infrared detector.
\end{abstract}

Keywords: silicon, infrared, photo detector.

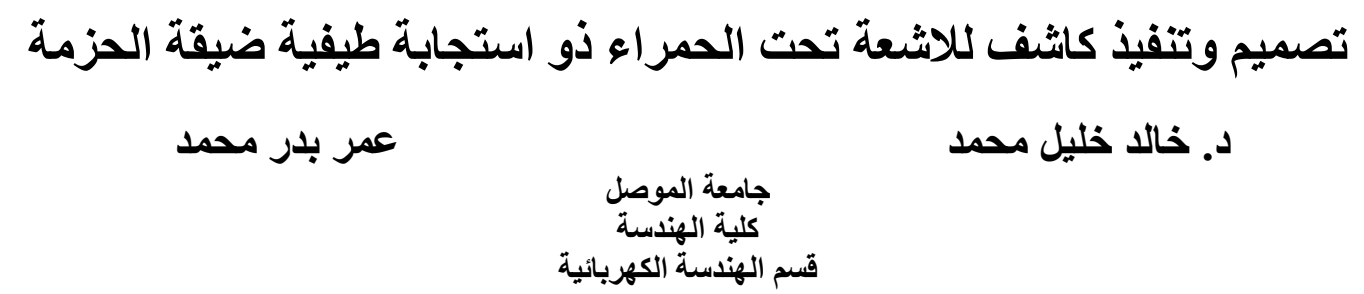

\footnotetext{
الخلاصة

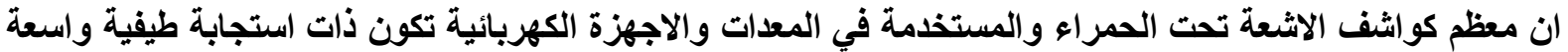

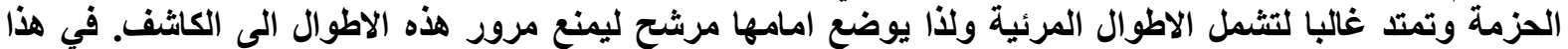

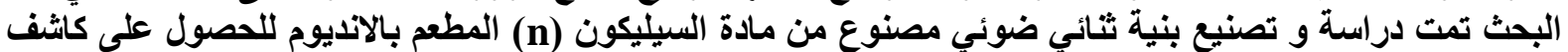

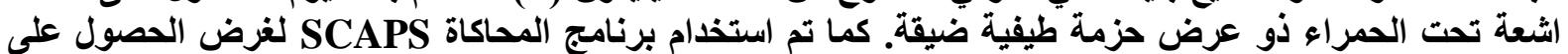

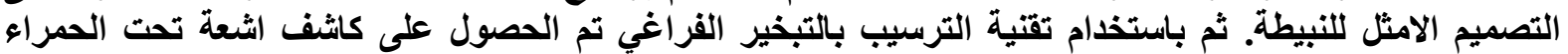

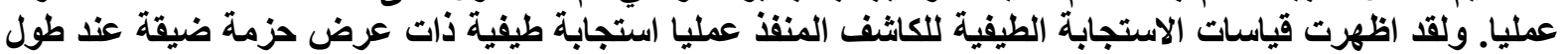

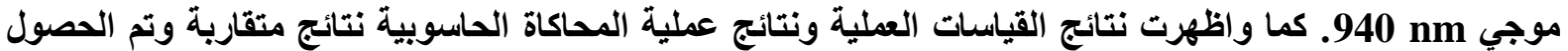
من خلالها على كاثف اشعة تحت حمراء بسيط ورخيص وذو عرض حزمة طيفية ضيقة.
} 


\section{1- Introduction}

Infrared remote control equipment needs to have narrowband spectral response so that its operation will not affected by visible light. Photo detectors are semiconductor devices that can detect optical signals through electronic processes. The operation of a general photo detector includes three processes: carrier generation by incident light, carrier transport, and extraction of carriers as terminal current to provide the output signal.

Photo detectors are important in optical-fiber communication systems operated in the near-infrared region $(0.8-1.6 \mu \mathrm{m})$. They demodulate optical signals, that is, convert the optical variations into electrical variations that are subsequently amplified and further processes. For such applications, the photo detectors must satisfy stringent requirements such as high sensitivity at operating wavelengths, high response speed, and minimum noise. In addition, the photo detector should be compact in size, uses low biasing voltage and current, and should be reliable under operating conditions [1].

\subsection{The Absorption of Light in Semiconductors}

Absorption occurs during the propagation of light through the medium if the frequency of the light is resonant with the transition frequencies of the atoms in the medium. In this case, the light will be attenuated as it progresses.

The absorption of light by an optical medium is quantified by its absorption coefficient $(\alpha)$; this is defined as the fraction of the power absorbed in a unit length of the medium. The absorption of a photon flux $\phi$ passes through a differential thickness element $d l$ are expressed by [2]:

$d \phi=-\alpha(\lambda) \phi d l$

With the solution for the remaining flux at depth $l$ being:

$\phi=\phi_{0} e^{-\alpha(\lambda) r}$

Where $\phi_{o}$ is the flux-entering detector. The absorption coefficient is a strong function of light wavelength, so that optical materials may absorb one color but not another.

Absorption coefficient not only determines whether light can be absorbed for photo excitation, but it also indicates where light is absorbed. A high value of absorption coefficient indicates that light is absorbed near the surface where light enters. A low value means that the absorption is low so that, light can penetrate deeper into the semiconductor. In the extreme, light is transparent for long wavelengths without photo excitation [1].

The total absorption coefficient in the detector's cell $\alpha_{\text {tot }}$ includes all the absorption processes, which are [3]:

$\alpha_{\text {tot }}(\lambda)=\alpha_{n}(\lambda)+\alpha_{p}(\lambda)+\alpha_{f c}(\lambda)+\alpha_{e-h}(\lambda)$

$\alpha_{n}(\lambda)=f_{t} N_{t} \sigma_{n}^{o p t}(\lambda)$

$\alpha_{p}(\lambda)=\left(1-f_{t}\right) N_{t} \sigma_{p}^{\text {opt }}(\lambda)$

$\alpha_{f c}(\lambda)=\left(2.7 \times 10^{-18} p+1.8 \times 10^{-18} n\right) \lambda^{2}$

Where:

$\alpha_{n}$ Is the absorption coefficient for electron photoemission from the IPV impurity,

$\alpha_{p}$ Represents the hole photoemission from the IPV impurity,

$\alpha_{f c}$ Is the absorption coefficient free carrier absorption, a process that does not result in the creation of electron-hole pairs.

$\alpha_{\theta-h}$ Is the absorption coefficient for intrinsic band-to-band electron-hole pair creation, 
$f_{t}$ Is the occupancy of the impurity level,

$N_{t}$ is the impurity concentration,

$\sigma_{n}^{\text {opt }}$ is the electron photoemission cross section of the impurity,

$\sigma_{p}^{o p t}$ is the hole photoemission cross section of the impurity,

$n$ and $p$ are the electron and hole concentration respectively, and

$\lambda$ is the free-space wavelength.

\subsection{Extrinsic Generation / Recombination Processes.}

Also called the modified Shockley Read Hall generation / recombination process, the six impurity-related transitions included in the modified SRH model are shown in figure (1). For steady state conditions, give the net recombination rate (U) via an impurity level [3]:

$$
U=\frac{n p-n_{1}^{*} p_{1}^{*}}{\tau_{n 0}\left(p+p_{1}^{*}\right)+\tau_{p 0}\left(n+n_{1}^{*}\right)}
$$

Where [3]:

$$
\begin{aligned}
& \tau_{n 0}=1 / c_{\mathrm{n}} \mathrm{N}_{\mathrm{t}} \\
& \tau_{p 0}=1 / \mathrm{c}_{\mathrm{p}} \mathrm{N}_{\mathrm{t}} \\
& n_{1}^{*}=n_{1}+\tau_{n 0} g_{n, \max } \\
& p_{1}^{*}=p_{1}+\tau_{p 0} g_{p, \max } \\
& n_{1}=g_{t} N_{c} e^{-\left(E_{\mathrm{c}}-E_{\mathrm{t}}\right) / k T} \\
& p_{1}=\frac{1}{g_{\mathrm{t}}} N_{v} e^{-\left(E_{\mathrm{t}}-E_{v}\right) / k T} \\
& g_{n, \max }=\mathrm{N}_{\mathrm{t}} \int_{\lambda_{g}}^{\lambda_{n, \max }} 2 \sigma_{\mathrm{n}}^{\text {opt }}(\lambda) \phi_{p h}(x, \lambda) d \lambda \\
& g_{p, \max }=\mathrm{N}_{\mathrm{t}} \int_{\lambda_{g}}^{\lambda_{p \max }} 2 \sigma_{\mathrm{p}}^{\text {opt }}(\lambda) \phi_{p h}(x, \lambda) d \lambda
\end{aligned}
$$

with the occupancy of the impurity level $\left(f_{t}\right)$ given by [3]:

$$
f_{t}=\frac{c_{n} n N_{t}+c_{p} p_{1} N_{t}+g_{p, \max }}{c_{n} N_{t}\left(n+n_{1}\right)+\left(g_{n, \max }+g_{p, \max }\right)+c_{p} N_{t}\left(p+p_{1}\right)}
$$

Equation (7) is widely applicable, no degeneracy being the major assumption. $\tau_{n 0}$ and $\tau_{p 0}$ are the SRH low-injection lifetimes for electrons and holes, $c_{\mathrm{n}}$ and $c_{\mathrm{p}}$ are the electron and hole capture coefficients, $g_{t}$ is the impurity-level degeneracy factor and $E_{t}$ the impurity-level energy, $E_{c}$ and $E_{v}$ are the conduction and valence band edges respectively, and $N_{c}$ and $N_{v}$ are the effective densities of states in the conduction and valence bands. $g_{n, \max }$ is the optical emission rate of electrons from the impurity, with the impurity fully occupied. $g_{p, \max }$ is the optical emission rate of holes from the impurity, with the impurity completely empty. $\sigma_{\mathrm{n}}^{\text {opt }}(\lambda)$ and $\sigma_{\mathrm{p}}^{\text {opt }}(\lambda)$ are the electron and hole photoemission cross sections of the impurity, with thresholds at $\lambda_{n, \max }$ and $\lambda_{p, \max }$ respectively. The lower limits on the wavelength integrations are assumed the silicon band gap wavelength $\lambda_{\mathrm{g}}=1.107 \mu \mathrm{m}$. It is assumed that all shorterwavelength photons are utilized by intrinsic band-to-band absorption. $\phi_{p h}(x, \lambda)$ is the photon flux density at a distance $x$ from the illuminated front surface. The factor 2 is a geometrical factor to account for the oblique passage of light within the textured cell considered. 
The lifetime arising from indirect transitions is inversely proportional to the trap density $\mathrm{N}_{\mathrm{t}}$. Many impurities have energy levels close to the middle of the band gap; these impurities are efficient recombination centers.

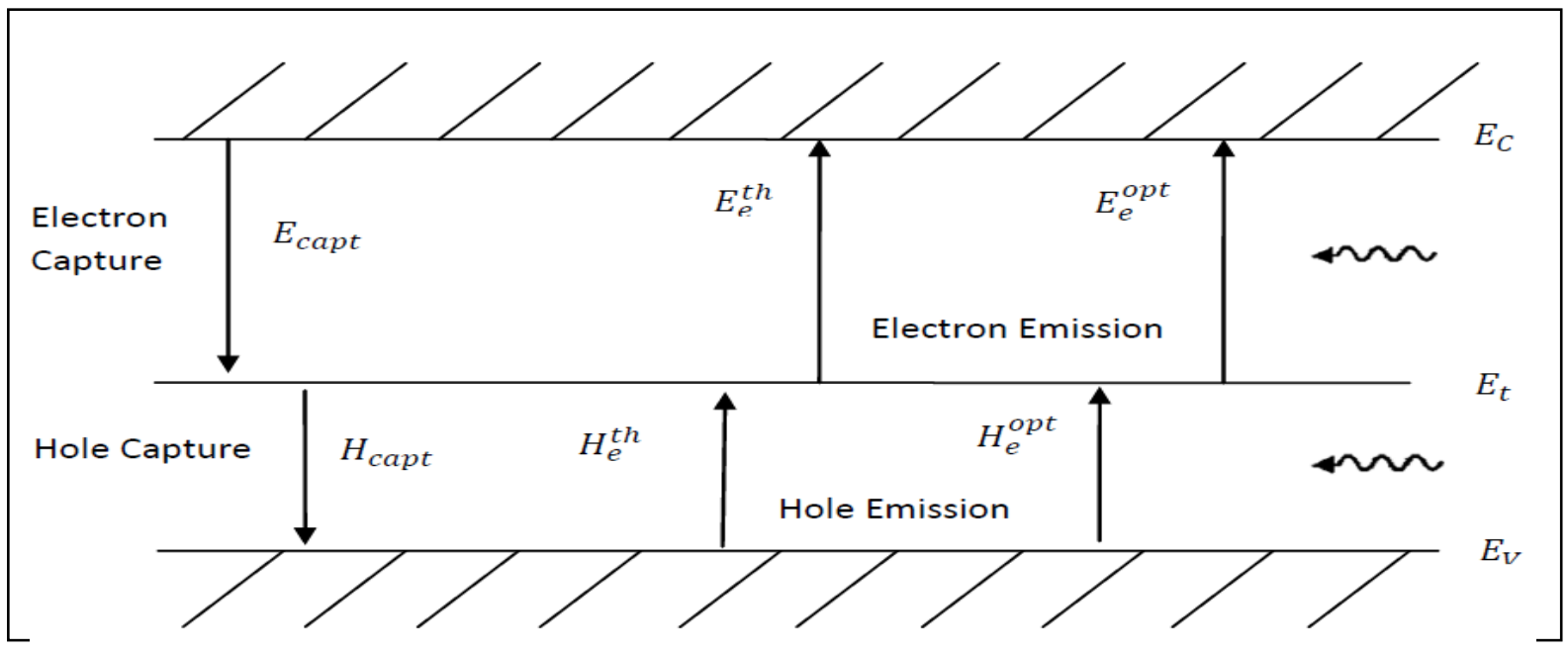

Figure (1): The six transitions of the modified SRH recombination model. Electron and hole emission can occur thermally (th) or optically (opt).

\subsection{Responsively and Quantum Efficiency}

Assume that within a certain observation period $t$ the photo detector has been struck by one photon with the energy $h v=h c / \lambda$. Then, the average optical radiation power is $p_{\text {opt }}=h v / t$. Ideally, this photon produces one electron-hole pair, which after separation supplies into the external circuit one electron with the charge $\mathrm{q}$, and thus the average current is $q / t$. The coefficient of conversion (the ratio of the output photocurrent $\left(I_{p}\right)$ to the incident optical radiation power $\left.\left(p_{\text {opt }}\right)\right)$ called the responsively of photo detector [4]:

$S_{R}(\lambda)=\frac{I_{p}}{p_{\text {opt }}}=\frac{q / t}{h v / t}=\frac{q}{h v}=\frac{q \lambda}{h c}$

Assume now that the ideal photo detector has received $\mathrm{N}$ photons within the time $\mathrm{t}$ .Then the photocurrent is increased $N$-fold $\left(I_{p}=N q / t\right)$, and so is the optical radiation power $\left(p_{\text {opt }}=N h v / t\right)$, but their ratio (responsively) remains unchanged.

In real photo detectors, however, some of the incident quanta are reflected, some other pass through the crystal without being absorbed, even some of the absorbed quanta are not lucky enough, and the electron-hole pairs generated by them recombine before reaching the junction. So it is only part of the incident quanta, $N^{\prime}=\eta N$ that produce pairs which are separated in the space charge region and cause photocurrent in the external circuit. It's noted that the photocurrent is only increased by the factor of $N^{\prime}$ and the expression for the responsively of real photo detectors contains the coefficient $\eta$ is [4]:

$S_{R}(\lambda)=\frac{I_{p}}{p_{\text {opt }}}=\frac{N^{\prime} q / t}{N h v / t}=\frac{N^{\prime}}{N} \frac{q}{h v}=\eta \frac{q \lambda}{h c}$

The coefficient $\eta$ characterizes the performance of photo detector as a counter of quanta of radiation, and is referred to as the quantum efficiency $(\mathrm{QE})$ of the photo detector 


\section{Infrared Detector Fabrication}

The fabricated samples are prepared by vacuum evaporation technique using Balzers unit (BA-510) as a coating system. The silicon (n) wafers are subjected to a rigorous cleaning cycle, in order to remove the native surface oxide layer and to reduce the pinholes formation. After the deposition of indium with the required thickness on the n-type silicon wafer, the samples are heated under vacuum to a temperature $350{ }^{\circ} \mathrm{C}$ to diffuse the indium deeply in silicon, with different periods of time the samples are subjected in order to achieve different junction depths. Aluminum thin films were deposited as back and front contacts for the fabricated samples. After the deposition of the aluminum film on the rough face of the silicon wafer the fabricated samples were heated under vacuum to a temperature of $350{ }^{\circ} \mathrm{C}$ for half an hour. This heat treatment is necessary to obtain an ohmic contact between the aluminum and the silicon wafers.

\section{Simulation Results}

The SCAPS program, which is, stands for Solar cell CAPacitance Simulator used as a simulation tool to analyze and achieve the optimum structure design. In principle, any numerical program capable of solving the basic semiconductor equations could be used for modeling thin film solar cells. The basic equations are, the Poisson equation, relating the charge to the electrostatic potential $(\psi)$, and the continuity equations for electrons and holes, and the electron and hole current density $I_{n}$ and $I_{p}$. Reference [5] gives the basic theoretical principles of this program.

\subsection{Simulation Parameter Values}

A substrate of silicon (n-type) is considered with appropriate silicon parameters such as energy gap, electron affinity, electron and hole mobility's etc. The incident light is from the p-layer. The optical cross section of an indium impurity in silicon for electrons and holes are given according to reference [3]. The surface transmission coefficient $T$ is assumed to be equal to 1 ; also reflectivity of back surface $R_{b}$ is chosen to be equal to 1 , which corresponds to best light trapping. The radiated recombination coefficient, Auger hole capture coefficient, and Auger hole capture coefficient are all assumed to be equal to 0. The absorption data for silicon are given in reference [6]. The capture cross section for electrons is $1 \times 10^{-22}$, and for holes is $5 \times 10^{-15}$ [7]. The investigation had been carried out for room temperature $\mathrm{T}=300 \mathrm{~K}$. The energy level of indium in silicon was accepted as $E_{t}=E_{v}+0.157 \mathrm{eV}$.

\subsection{Effect of Indium Concentration on the Spectral Response}

Figure (2) shows the increase of QE with indium concentration increasing. This is because of two effects working together; the increase of electric field strength in the depletion region and the total absorption coefficient increasing due to the indium concentration increasing.

When the indium concentration is below the background concentration by a significant amount, the QE of the photodiode is similar to that of a piece of silicon doped with one type of shallow impurities, and this is because there is no junction formed yet. Figure (2) curve (a) shows this case. 


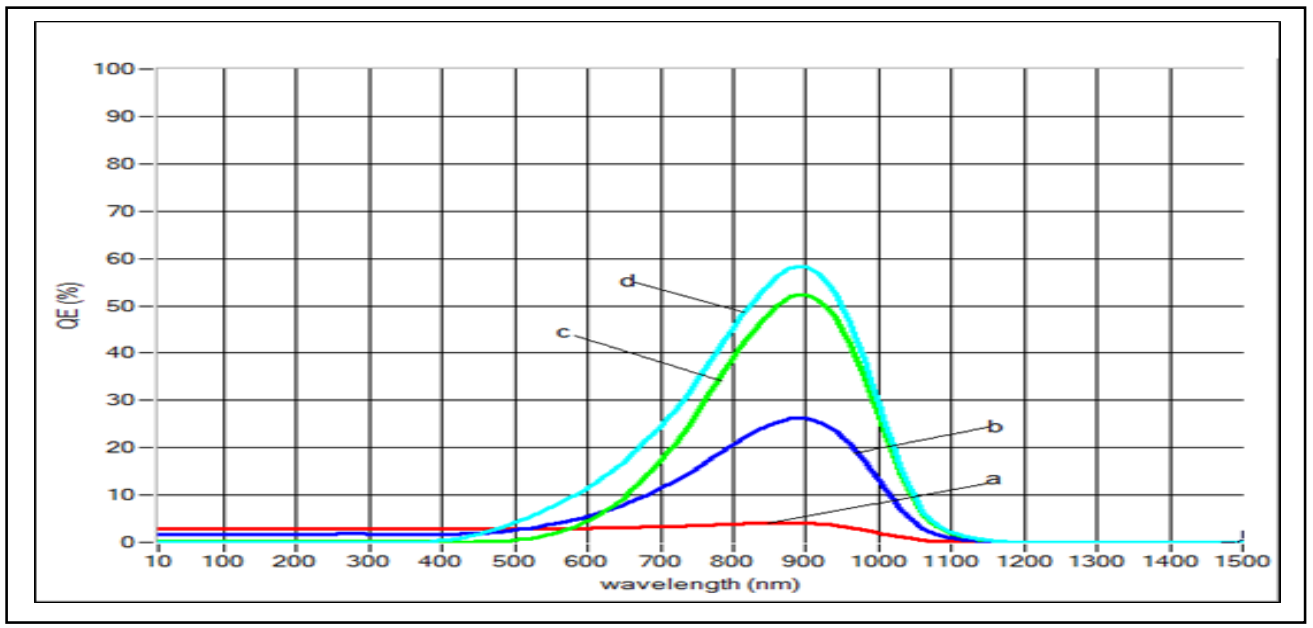

Figure (2): The effect of indium concentration a- $1 \times 10^{13}, \mathrm{~b}-8 \times 10^{13}, \mathrm{c}-1 \times 10^{14}$, d$1 \times 10^{18} / \mathrm{cm}^{3}$. With background concentration equal to $1 \times 10^{14}$ and $\mathrm{Th}_{\mathrm{n}}=100 \mu \mathrm{m}, \mathrm{Th}_{\mathrm{p}}=20 \mu \mathrm{m}$.

Once the junction formed with sufficient indium concentration (determined by background concentration), as the indium concentration increases, the electric field strength in the depletion region also increase, which causes more photo generated charge carriers to contribute in the generation of photocurrent.

Also the increase of indium concentration will be responsible for total absorption coefficient increment through the increasing of absorption coefficient of electron and hole photoemission from the impurity levels.

\subsection{Effect of N-Region Doping Level on the Spectral Response}

The dopant type in the n-region is shallow dopant, and figure (3) shows the effect of doping level in the $\mathrm{n}$-region on the spectral response. As the case of indium, the shallow dopant increment will increase the depletion region electric field strength, which results in more charge carriers to contribute in photocurrent generation.

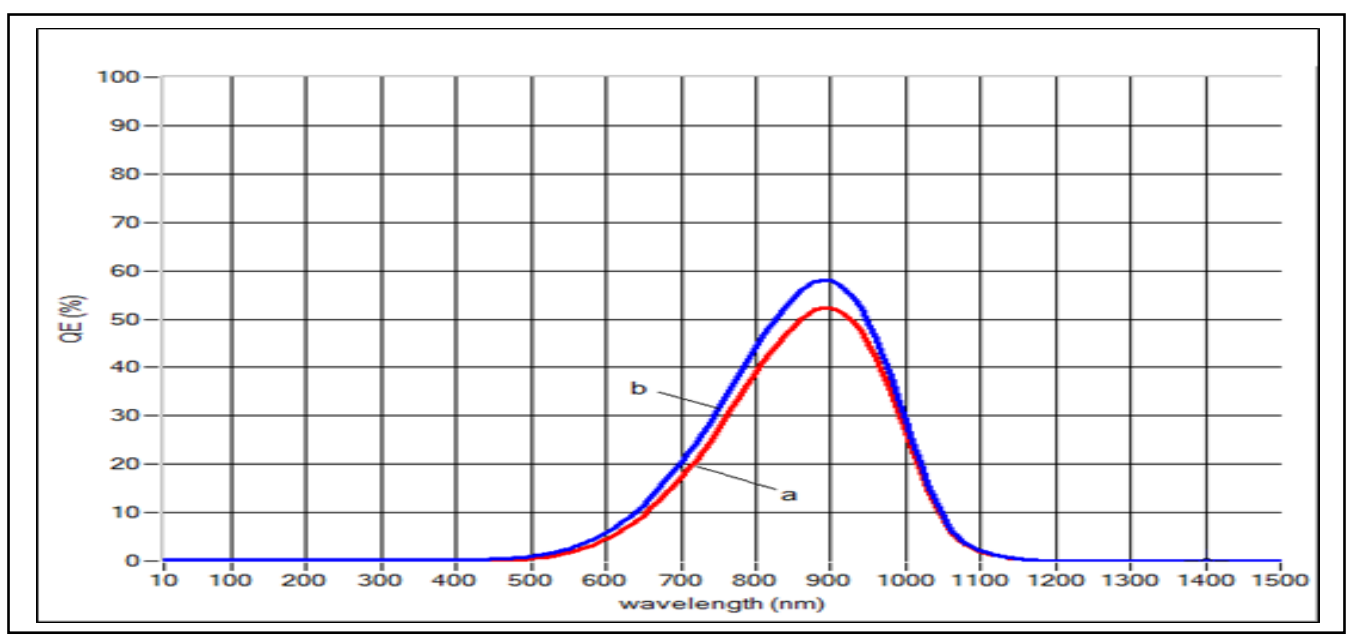

Figure (3): The effect of n-region concentration a- $1 \times 10^{14}$, b- $1 \times 10^{16} / \mathrm{cm}^{3}$. With $n_{p}$ dopant $10^{14}$ and indium concentration $10^{14}, T h_{n}=100 \mu m, T h_{p}=20 \mu m$. 
Also by a slight contribution, the increase of shallow dopant concentration will results in increasing total absorption coefficient but this contribution has much little effect than in indium case and that is because the dopant is shallow level, which means inefficient generation- recombination centers.

\subsection{P-Region Dopant Type Effect on the Spectral Response}

In figure (4) a comparison between two types of dopant in the p-region; indium which is a medium-deep level dopant and shallow-level dopant. Two differences exist: the indium dopant photodiode has narrower spectral response and smaller QE than shallow-dopant photodiode. The medium-deep level dopants are more efficient generation-recombination centers compared with the shallow dopant, therefore; those medium-deep level dopant makes the lifetime of electrons and holes photogeneratedly in the photodiode smaller, and becomes much smaller as the concentration of those dopant increase.

The smaller lifetime of medium-deep level photodiode compared to that of shallowlevel photodiode will cause less of the photogeneratedly carriers to reach the depletion region and contribute in photocurrent generation, because of the increasing rate of recombination.

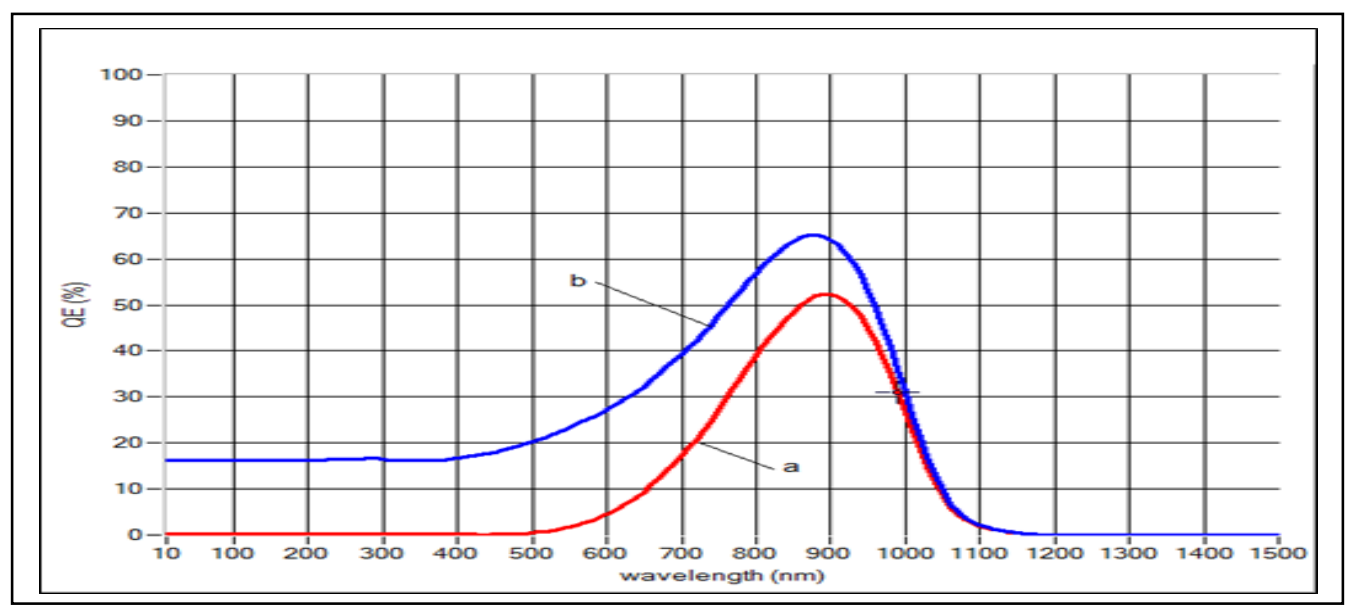

Figure (4): a- medium-deep level (indium) dopant, b- shallow-level dopant. Silicon substrate with $\mathrm{Th}_{\mathrm{n}}=100 \mu \mathrm{m}, \mathrm{Th}_{\mathrm{p}}=20 \mu \mathrm{m}$ and background concentration $10^{14}$.

\subsection{Junction Depth Effect on the Spectral Response}

At any junction depth there is a wavelength at which maximum QE occurs, it denoted by $\eta_{\max }$ this is because of at that wavelength, there is maximum carrier collection in the depletion region. This case can be observed in any curve on figures (5) and (6).

Also, at any doping level, there is a junction depth at which maximum $\eta_{\max }$ occurs. As can be noted from figures $4.2,4.3$ at doping level $10^{14}$, the junction depth of $20 \mu \mathrm{m}$ the maximum $\eta_{\max }$ occurs, and at doping level $10^{16}$, the junction depth is $5 \mu \mathrm{m}$.

It can be noted two important things, those are; as doping level increases, the junction depth at which maximum $\eta_{\max }$ occurs decreases. Also, the value of maximum $\eta_{\max }$ increases. The reason of those are; as the doping level increases the carrier lifetime will decreases, so, in order to maximize the QE to maximum $\eta_{\max }$ then it must bring the depletion region closer to the surface so as to achieve maximum carrier collection in the depletion region. The bringing of the depletion region more close to the surface from one side, which cause more carrier generation happened in the depletion region, and the increasing of absorption coefficient due to increasing of doping level from the other side, will cause the photocurrent to be increased and hence the QE will increase. 
The increasing of junction depth results in narrower spectral response because shorter wavelengths will generate carriers in a region which is far from the depletion region so that the time required to reach the depletion region is larger than the carrier lifetime, so that they recombine before reaching the depletion region and causing the spectral response move toward longer wavelengths.

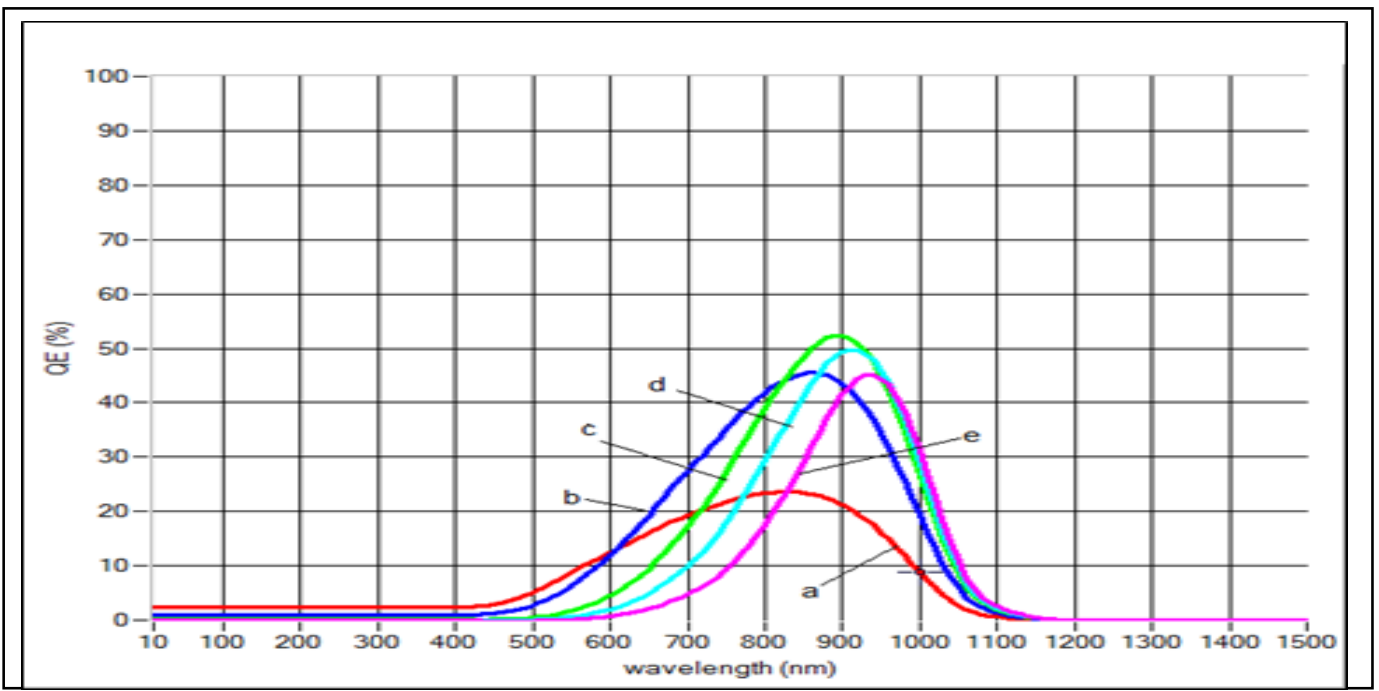

Figure (5): effect of Junction depth a- $5 \mu \mathrm{m}, \mathrm{b}-10 \mu \mathrm{m}, \mathrm{c}-20 \mu \mathrm{m}, \mathrm{d}-30 \mu \mathrm{m}, \mathrm{e}-50 \mu \mathrm{m}$. with background and indium concentration equal to $10^{14}$ and $\mathrm{Th}_{\mathrm{n}}=100 \mu \mathrm{m}$.

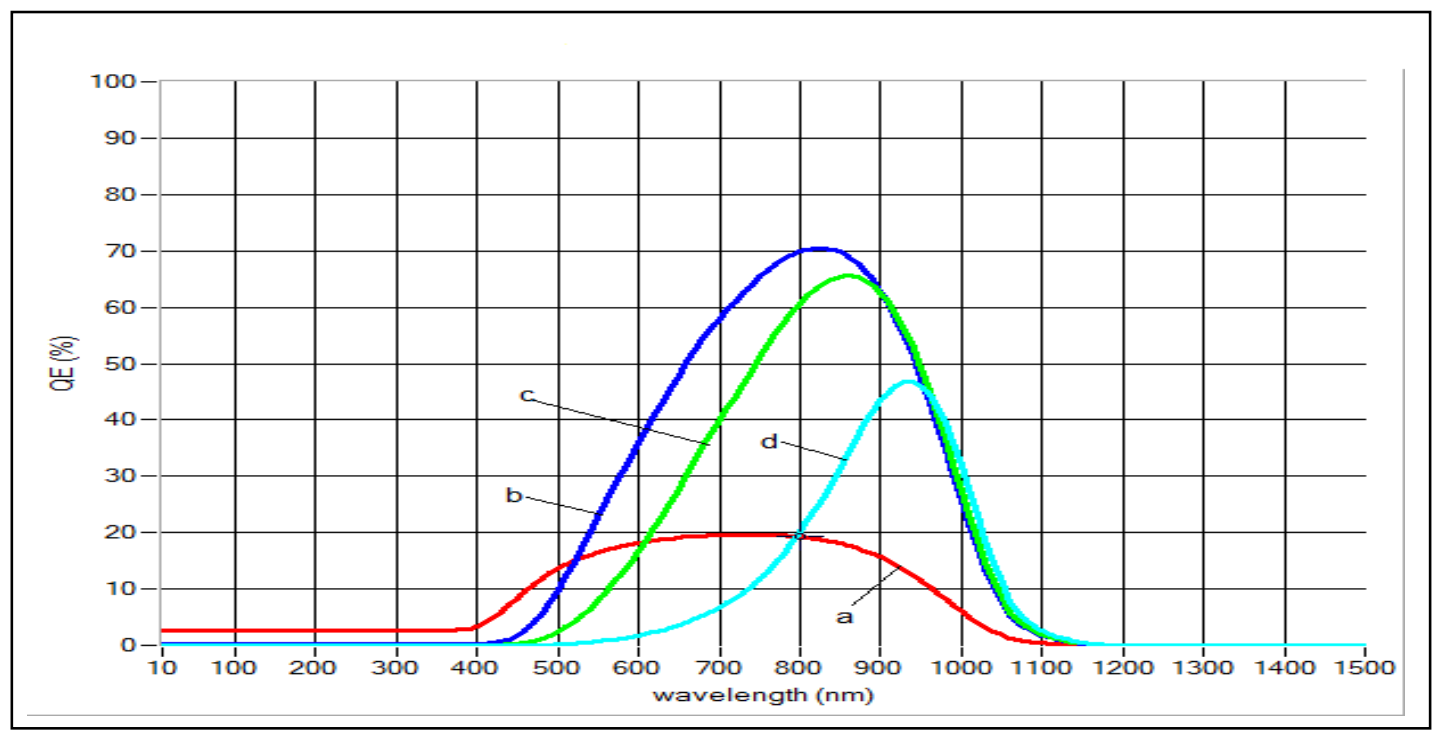

Figure (6): effect of junction depth a- $1 \mu \mathrm{m}, \mathrm{b}-5 \mu \mathrm{m}$, c- $10 \mu \mathrm{m}$, d- $50 \mu \mathrm{m}$. With background and indium concentration equal to $10^{16}$ and $T h_{n}=100 \mu m$.

\subsection{N-Region Length Effect on the Spectral Response}

As the n-region length increases, the QE curves move to higher levels and the spectral response becomes wider, this is shown in figure (7). It can be say that as the n-region length increases, the longer wavelengths of light incident, will take the chance to generate carriers, especially if it knows that at those wavelengths the absorption coefficient becomes small, which in turns need to travel longer distances in order that the absorption process to take 
place. So it can be seen from figure (7) that as the n-region length becomes wider, those long wavelengths generate more carriers and therefore the QE curves become higher at those wavelengths.

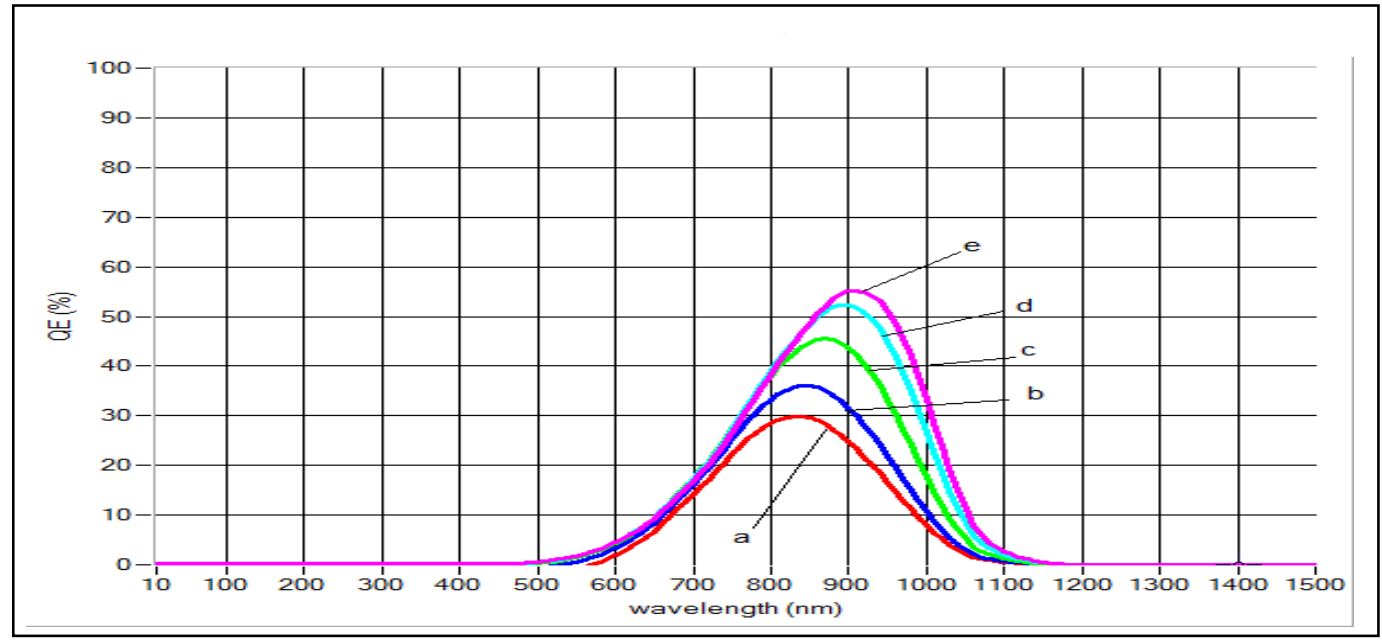

Figure (7): effect of n-region length a- $10 \mu \mathrm{m}$, b- $20 \mu \mathrm{m}$, c- $50 \mu \mathrm{m}, \mathrm{d}-100 \mu \mathrm{m}$,e- $150 \mu \mathrm{m}$. With background and indium concentration equal to $10^{14}$ and $T h_{p}=20 \mu \mathrm{m}$.

\section{Practical Results and Discussion}

As discussed earlier in section (V) for structure fabrication, the practical sample is fabricated according to it, and figure (8) shows a block diagram of the fabricated photodiode. The fabricated photodiode has approximately the following parameter values:

$$
\begin{aligned}
& N_{D}=10^{14} / \mathrm{cm}^{3} \\
& N_{A}=10^{15} / \mathrm{cm}^{3} \\
& V_{b i}=0.5 \mathrm{volt} \\
& x_{n}=57 \mathrm{~nm} \\
& x_{n}=0.54 \mathrm{~nm}
\end{aligned}
$$

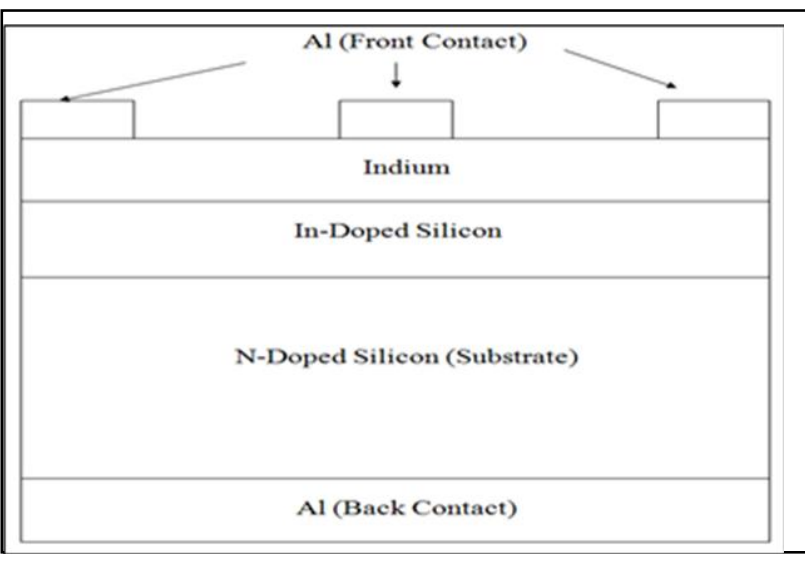

Figure (8) photodiode Block Diagram

Figure (9) and (10) shows the current response and the relative quantum efficiency of the infrared photodiode respectively. The short circuit current was measured at 4000 lux and found that the maximum current can be equal to $6 \mu \mathrm{A}$ at $941.1 \mathrm{~nm}$ wavelength. It is clear that at the junction depth formed, the electron-hole pairs generated are almost collected inside the junction yielding a maximum current generation.

From figure (9), there is no photoresponse of the photodiode at wavelengths less than $450 \mathrm{~nm}$. This can be attributed to the high absorption coefficients at these wavelengths so that all generation-recombination processes take place near the surface and the generated electron-hole pairs recombine before reach the depletion layer. 
The current starts increasing at $\lambda>450 \mathrm{~nm}$ and the maximum current obtained at wavelength $941.1 \mathrm{~nm}$. This can be explained by the fact that at this region of photoresponse, the two semiconducting layers of the photodiode (n-type silicon and p-type indium-doped silicon) and the depletion layer contribute in the photogeneration of the electron-hole pairs.

As $\lambda$ increases above $941.1 \mathrm{~nm}$; the current starts decreasing gradually, the current generation at this range can be attributed to the fact that at this range of wavelengths most of the generated electron-hole pairs are generated due to subgap absorption mechanism (External SRH generation-recombination process) which extend the photodiode response up to $1100 \mathrm{~nm}$ or more, the energy of the photons of the light which have wavelength longer than $1100 \mathrm{~nm}$ are so small such that there is no enough energy capable of lifting the electrons to conduction band and hence no photoresponse.

The effect of wavelength on the photocurrent response can be used to interpret the effect of wavelength on the quantum efficiency and responsivity which was related by equations (17) and (18).

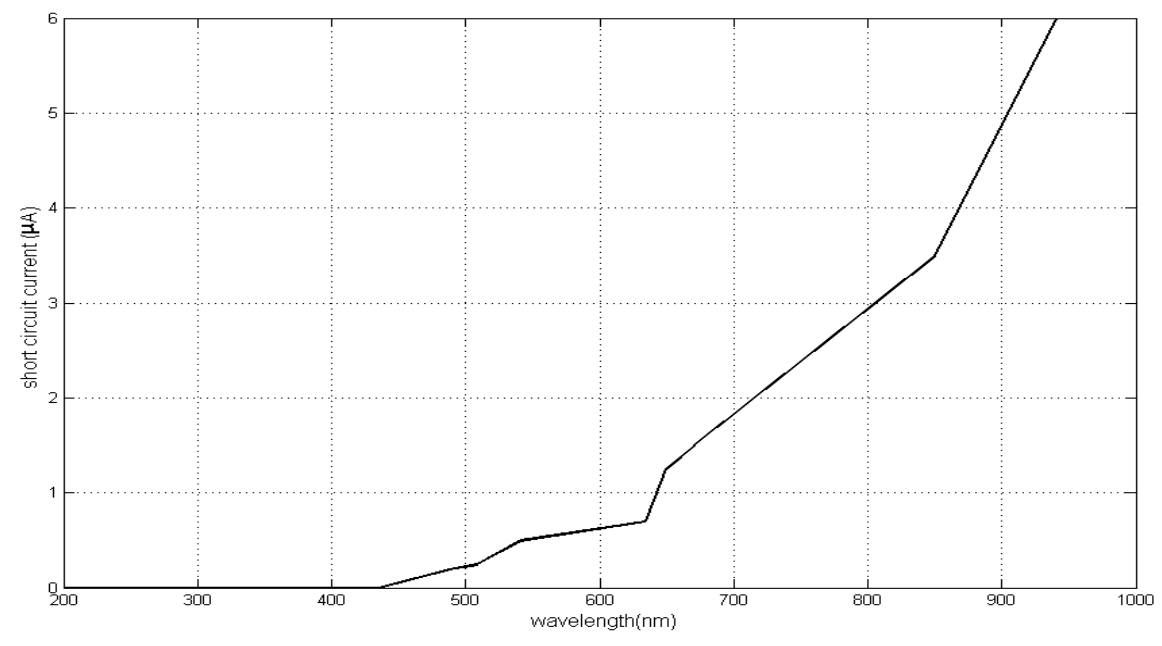

Figure (9): current spectral response

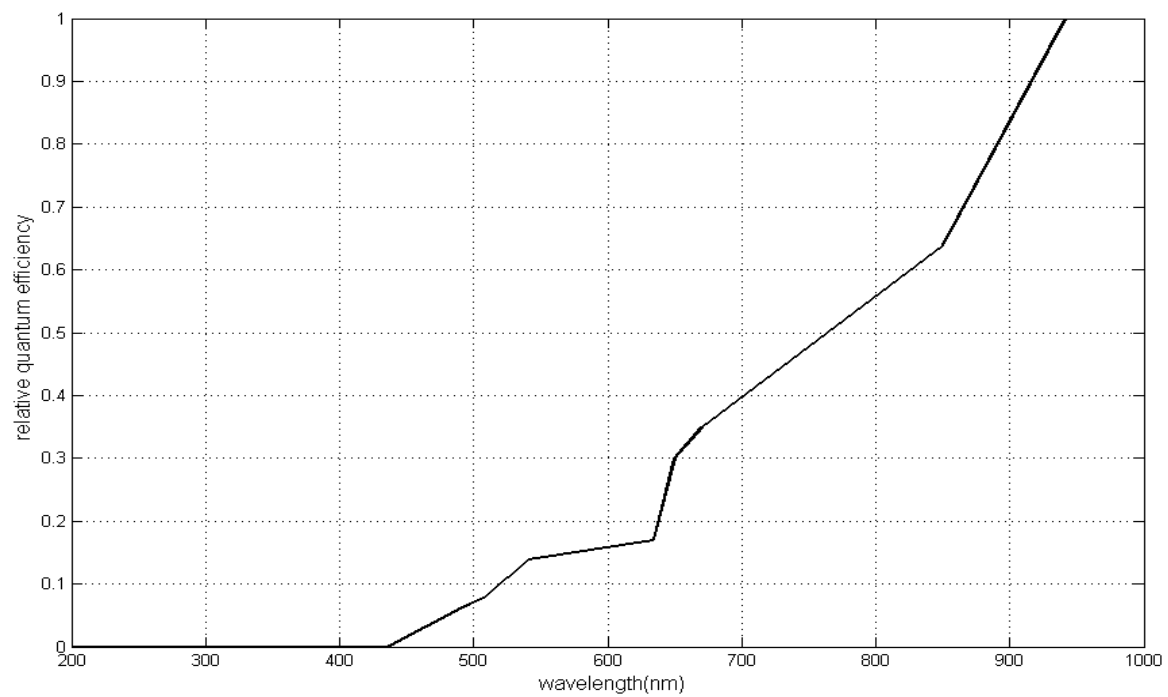

Figure (10): photodetector relative quantum efficiency 


\section{Conclusion}

It is noted that by changing the indium concentration, the junction depth at which the maximum quantum efficiency occurs is changing also, and as the indium concentration increases the junction depth at which the maximum quantum efficiency occurs decreases. The increase of the junction depth results in decrease of the range of spectral response of the photodiode. Also, as the generation-recombination centers become more efficient as recombination center, by replacing the shallow dopants by the indium, then the spectral response range will be narrower and move toward the longer wavelengths. This study could be utilized in the silicon-based photodetectors manufacturing.

\section{References}

[1] S. M. Sze, Kwok K. Ng, "Physics of Semiconductor Devices" $3^{\text {rd }}$ Ed., JOHN WILEY \& SONS INC., 2007. P: 663-664.

[2] Mark Fox, "Optical Properties of Solids", Oxford University Press, 2001. P: 2-3.

[3] M. J. Keevers, M. A. Green, "Efficiency improvements of silicon solar cells by the Impurity photovoltaic effect", J. Appl. Phys. 75 (8), 15 April 1994. P: 4022-4031.

[4] M. A. Trishenkov, "Detection of Low-Level Optical Signals, Photodetectors, Focal Plane Arrays and Systems", Kluwer Academic Publishers, 1997. P: 23-24.

[5] Alex Niemegeers, Sofie Gillis, Marc Burgelman, "A user program for realistic simulation of polycrystalline heterojunction solar cells: SCAPS-1D", $2^{\text {nd }}$ World Conference on Photovoltaic Energy Conversion, 1998. pp. 672-675.

[6] Martin A. Green " Silicon Solar Cells Advanced Principles \& Practice", pp.333-336.

[7] M. Schmeits and A. A. Mani, " Impurity photovoltaic effect in c -Si solar cells. A numerical study ", Journal of Applied Physics Volume 85, number 4, 15 February 1999, p: 2207-2212. 